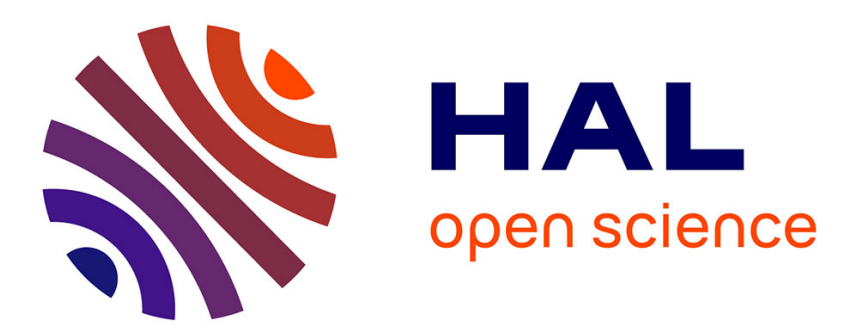

\title{
Variance Reduction for Generalized Likelihood Ratio Method in Quantile Sensitivity Estimation
} Yijie Peng, Michael C Fu, Jiaqiao Hu, Pierre L'Ecuyer, Bruno Tuffin

\section{To cite this version:}

Yijie Peng, Michael C Fu, Jiaqiao Hu, Pierre L'Ecuyer, Bruno Tuffin. Variance Reduction for Generalized Likelihood Ratio Method in Quantile Sensitivity Estimation. 2021 - Winter Simulation Conference, Dec 2021, Phoenix, United States. pp.1-12. hal-03196364

\section{HAL Id: hal-03196364 https://hal.inria.fr/hal-03196364}

Submitted on 12 Apr 2021

HAL is a multi-disciplinary open access archive for the deposit and dissemination of scientific research documents, whether they are published or not. The documents may come from teaching and research institutions in France or abroad, or from public or private research centers.
L'archive ouverte pluridisciplinaire HAL, est destinée au dépôt et à la diffusion de documents scientifiques de niveau recherche, publiés ou non, émanant des établissements d'enseignement et de recherche français ou étrangers, des laboratoires publics ou privés. 


\title{
Variance Reduction for Generalized Likelihood Ratio Method in Quantile Sensitivity Estimation
}

\author{
Yijie Peng \\ Guanghua School of Management \\ Peking Universtiy \\ Beijing 100871, China
}

Jiaqiao $\mathrm{Hu}$

Dept. of Applied Mathematics \& Statistics

Stony Brook University

Stony Brook, NY 11794-3600, USA

\author{
Michael C. Fu \\ The Robert H. Smith School of Business \\ Universtity of Maryland \\ College Park, Maryland 20742, USA
}

Pierre L'Ecuyer

Dept. of Computer Science \& Operations Research

University of Montreal

Montreal, Quebec, Canada

\author{
Bruno Tuffin \\ INRIA, Univ Rennes, CNRS, IRISA \\ Campus de Beaulieu, 35042 Rennes Cedex, France
}

\begin{abstract}
We apply the generalized likelihood ratio (GLR) methods in Peng et al. (2018) and Peng et al. (2021) to estimate quantile sensitivities. Conditional Monte Carlo and randomized quasi-Monte Carlo methods are used to reduce the variance of the GLR estimators. The proposed methods are applied to a toy example and a stochastic activity network example. Numerical results show that the variance reduction is significant.
\end{abstract}

\section{INTRODUCTION}

Quantile, also known as value-at-risk (VaR), is an important risk measure. Quantile sensitivity estimation has been actively studied in simulation due to its centrality in risk management. A seminal work is Hong (2009), which proposes a conditional infinitesimal perturbation analysis (IPA). Jiang and Fu (2015) avoid the need of computing the conditional expectation for some special cases. Liu and Hong (2009) propose a kernel-based estimator for estimating the quantile sensitivity, which can be extended to estimate sensitivities of conditional VaR (CVaR) (Hong and Liu 2009). A conditional Monte Carlo (CMC) method is applied to estimate quantile sensitivity in Fu et al. (2009). Heidergott and Volk-Makarewicz (2016) propose a measure-valued differentiation (MVD) estimator for quantile sensitivity. CMC and MVD for quantile sensitivity rely on estimating two distribution sensitivities, i.e., the derivatives of the distribution function with respect to both argument and parameters in the stochastic model.

The difficulty in distribution sensitivity estimation lies in the discontinuity introduced by the indicator function. Recently, Peng et al. (2018) propose a generalized likelihood ratio (GLR) method to handle discontinuities for a wide scope of sample performance in sensitivity analysis. Peng et al. (2020) develop GLR estimators for any distribution sensitivity and use it to calculate maximum likelihood estimation for complex stochastic models. Peng et al. (2021) derive a GLR estimator for stochastic models with uniform random numbers as input, relaxing a requirement in Peng et al. (2018) that the tails of the input distribution of the stochastic model go smoothly to zero fast enough. Peng et al. (2017) apply GLR to estimate quantile sensitivity, and Peng et al. (2019) extend the GLR quantile sensitivity estimator to stochastic model with 
correlations and jumps. Glynn et al. (2021) use the GLR estimators for distribution sensitivities to estimate derivatives of distortion risk measures, which cover VaR and CVaR as special cases.

CMC methods can reduce the variance and smooth the performance function in simulation by conditioning on certain events or random variables and then integrating out the remaining randomness (Asmussen and Glynn 2007). Fu et al. (2009) uses CMC to smooth the discontinuity introduced by the indicator function, after which IPA is applied to differentiate the conditional expectation. GLR does not need smoothing to obtain an unbiased distribution sensitivity estimator, but CMC can be applied afterward to reduce the variance for GLR.

As other variance reduction techniques, RQMC methods replace the vectors of uniform random numbers that drive independent simulation runs by dependent vectors of uniform random numbers that cover the space more evenly. When estimating an expectation, they can provide an unbiased estimator with its variance converging to zero at a rate that is faster than with Monte Carlo (Lemieux 2009; Dick and Pillichshammer 2010). Such a faster rate can be proved when the estimator inside the expectation is sufficiently smooth as a function of the underlying uniform random numbers. When the estimator is not smooth (e.g., discontinuous), the convergence rate may not be improved, but RQMC could still reduce the variance by a constant factor. We show, through simulation experiments, that the variance of the quantile sensitivity estimator by GLR can be significantly reduced by appropriately combining the method with CMC and RQMC. Similar use of CMC and RQMC for reducing the variance of quantile estimation can be found in Nakayama et al. (2020).

The rest of the paper is organized as follows. Section 2 introduces the quantile sensitivity estimation problem and the GLR method. Variance reduction by CMC and RQMC is discussed in Section 3. Section 4 provide applications and simulation experiments. The last section offers conclusions.

\section{QUANTILE SENSITIVITY ESTIMATION}

For $0 \leq \alpha \leq 1$, the $\alpha$-quantile (also known as value-at-risk) of an output random variable $Y_{\theta}=h(X ; \theta)$ with input random variables $X=\left(X_{1}, \ldots, X_{n}\right)$ and cumulative distribution function (cdf) $F(\cdot ; \theta)$ is defined as

$$
q_{\alpha}(\theta):=\arg \inf \{y: F(y ; \theta) \geq \alpha\} .
$$

When $F(\cdot ; \theta)$ is continuous, $q_{\alpha}(\theta)=F^{-1}(\alpha ; \theta)$. Our goal is to estimate the derivative of the $\alpha$-quantile with respect to parameter $\theta, d q_{\alpha}(\theta) / d \theta$. Assume $h(X ; \theta)$ is a continuous r.v. with a positive and continuous density $f(y ; \theta)$ on $\left(q_{\alpha}(\theta)-\varepsilon, q_{\alpha}(\theta)+\varepsilon\right), \varepsilon>0$. Using the formula for the derivative of an implicit function, we have (Fu et al. 2009)

$$
\frac{d}{d \theta} q_{\alpha}(\theta)=-\left.\frac{\partial F(y ; \theta)}{\partial \theta}\right|_{y=q_{\alpha}(\theta)} / f\left(q_{\alpha}(\theta) ; \theta\right) .
$$

Notice that

$$
\frac{\partial F(y ; \theta)}{\partial \theta}=\frac{\partial \mathbb{E}[\mathbf{1}\{h(X ; \theta) \leq y\}]}{\partial \theta}, \quad f(y ; \theta)=\frac{\partial \mathbb{E}[\mathbf{1}\{h(X ; \theta) \leq y\}]}{\partial y} .
$$

From Peng et al. (2020), we have the GLR estimators for these distribution sensitivities: $G_{1, i}(X ; y, \theta):=$ $\mathbf{1}\{h(X ; \theta) \leq y\} \psi_{1, i}(X ; \theta)$ and $G_{2, i}(X ; y, \theta):=\mathbf{1}\{h(X ; \theta) \leq y\} \psi_{2, i}(X ; \theta), i=1, \ldots, n$, such that

$$
\frac{\partial F(y ; \theta)}{\partial \theta}=\mathbb{E}\left[G_{1, i}(X ; y, \theta)\right], \quad f(y ; \theta)=\mathbb{E}\left[G_{2, i}(X ; y, \theta)\right],
$$


where

$$
\begin{aligned}
\psi_{1, i}(x ; \theta) & =\frac{\partial \log f_{X}(x ; \theta)}{\partial \theta} \\
& -\left(\frac{\partial h(x ; \theta)}{\partial x_{i}}\right)^{-1}\left[\frac{\partial h(x ; \theta)}{\partial \theta}\left(\frac{\partial \log f_{X}(x ; \theta)}{\partial x_{i}}-\frac{\partial^{2} h(x ; \theta)}{\partial x_{i}^{2}}\left(\frac{\partial h(x ; \theta)}{\partial x_{i}}\right)^{-1}\right)+\frac{\partial^{2} h(x ; \theta)}{\partial \theta \partial x_{i}}\right], \\
\psi_{2, i}(x ; \theta) & =\left(\frac{\partial h(x ; \theta)}{\partial x_{i}}\right)^{-1}\left(\frac{\partial \log f_{X}(x ; \theta)}{\partial x_{i}}-\frac{\partial^{2} h(x ; \theta)}{\partial x_{i}^{2}}\left(\frac{\partial h(x ; \theta)}{\partial x_{i}}\right)^{-1}\right),
\end{aligned}
$$

with $f_{X}(\cdot ; \theta)$ being the (joint) density of the vector of input random variables $X$. From Peng et al. (2021), we have the GLR estimators for distribution sensitivities when $X$ is a vector of uniform random numbers $U=\left(U_{1}, \ldots, U_{n}\right)$ :

$\widetilde{G}_{1, i}(U ; y, \theta):=\mathbf{1}\left\{h\left(\bar{U}_{i} ; \theta\right)-y \leq 0\right\} r_{i}\left(\bar{U}_{i} ; \theta\right)-\mathbf{1}\left\{h\left(\underline{U}_{i} ; \theta\right)-y \leq 0\right\} r_{i}\left(\underline{U}_{i} ; \boldsymbol{\theta}\right)+\mathbf{1}\{h(U ; \theta)-y \leq 0\} d(U ; \theta)$,

where

$$
\begin{aligned}
& r_{i}(u ; \theta)=\left(\frac{\partial h(u ; \theta)}{\partial u_{i}}\right)^{-1} \frac{\partial h(u ; \theta)}{\partial \theta} \\
& d(u ; \theta)=\left(\frac{\partial h(u ; \theta)}{\partial u_{i}}\right)^{-1}\left[\left(\frac{\partial h(u ; \theta)}{\partial u_{i}}\right)^{-1} \frac{\partial h(u ; \theta)}{\partial \theta} \frac{\partial^{2} h(u ; \theta)}{\partial u_{i}^{2}}-\frac{\partial^{2} h(u ; \theta)}{\partial u_{i} \partial \theta}\right],
\end{aligned}
$$

and

$$
\widetilde{G}_{2, i}(U ; z, \theta):=\mathbf{1}\left\{h\left(\bar{U}_{i} ; \theta\right)-y \leq 0\right\} \tilde{r}_{i}\left(\bar{U}_{i} ; \theta\right)-\mathbf{1}\left\{h\left(\underline{U}_{i} ; \theta\right)-y \leq 0\right\} \tilde{r}_{i}\left(\underline{U}_{i} ; \theta\right)+\mathbf{1}\{h(U ; \theta)-y \leq 0\} \tilde{d}(U ; \theta),
$$

where $\bar{U}_{i}:=(U_{1}, \ldots, \underbrace{1^{-}}_{i \text { th element }}, \ldots, U_{n}), \underline{U}_{i}:=(U_{1}, \ldots, \underbrace{0^{+}}_{i \text { th element }}, \ldots, U_{n})$, and

$$
\tilde{r}_{i}(u ; \theta)=-\left(\frac{\partial h(u ; \theta)}{\partial u_{i}}\right)^{-1} \quad \tilde{d}(u ; \theta)=-\left(\frac{\partial h(u ; \theta)}{\partial u_{i}}\right)^{-2} \frac{\partial^{2} h(u ; \theta)}{\partial u_{i}^{2}} .
$$

Let $X^{(j)}, j=1, \ldots, m$, be i.i.d. realizations of $X$, and $\hat{F}_{m}(\cdot)$ be the empirical distribution of $h\left(X^{(j)} ; \theta\right)$, $j=1, \ldots, m$. The empirical $\alpha$-quantile $\hat{F}_{m}^{-1}(\alpha)$ is the inverse of the empirical cdf evaluated at $\alpha$, defined as in (1) with $F$ replaced by $\hat{F}_{m}^{-1}$. Then the quantile sensitivity can be estimated by

$$
-\left.\frac{\sum_{j=1}^{m} \mathbf{1}\left\{h\left(X^{(j)} ; \theta\right) \leq y\right\} \psi_{1, i}\left(X^{(j)} ; \theta\right)}{\sum_{j=1}^{m} \mathbf{1}\left\{h\left(X^{(j)} ; \theta\right) \leq y\right\} \psi_{2, i}\left(X^{(j)} ; \theta\right)}\right|_{y=\hat{F}_{m}^{-1}(\alpha)} .
$$

This is a random function of a ratio of expectations by a ratio of averages evaluated at a random estimate. Even if each term in the ratio (2) is an unbiased estimator of the corresponding expectation, the ratio (2) is not an unbiased estimator of the ratio of expectations. There is also another source of bias for the sensitivity: the quantile estimator itself is also biased. Also, the two terms in (2) and quantile estimators are not independent because they are estimated by a same batch of inputs and outputs: $X^{(j)}$ and $h\left(X^{(j)} ; \theta\right)$, $j=1, \ldots, m$. The strong consistency and a central limit theorem for (2) have been established in Peng et al. (2017) using an empirical process theory.

Peng et al. (2018) consider sensitivity analysis for more general stochastic models:

$$
\frac{\partial}{\partial \theta} \mathbb{E}[\varphi(g(X ; \theta))]
$$


where $\varphi: \mathbb{R}^{n} \rightarrow \mathbb{R}$ is a measurable function not necessarily continuous, $g(x ; \theta)=\left(g_{1}(x ; \theta), \ldots, g_{n}(x ; \theta)\right)^{T}$ is a vector of functions with sufficient smoothness for $x \in \mathbb{R}^{n}$, and $X=\left(X_{1}, \ldots, X_{n}\right)$ is a vector of input random variables with a joint density $f_{X}(x ; \theta)$ supported on $\Omega \subseteq \mathbb{R}^{n}$. The Jacobian of $g(\cdot ; \theta)$ is

$$
J_{g}(x ; \theta):=\left(\begin{array}{cccc}
\frac{\partial g_{1}(x ; \theta)}{\partial x_{1}} & \frac{\partial g_{1}(x ; \theta)}{\partial x_{2}} & \ldots & \frac{\partial g_{1}(x ; \theta)}{\partial x_{n}} \\
\frac{\partial g_{2}(x ; \theta)}{\partial x_{1}} & \frac{\partial g_{2}(x ; \theta)}{\partial x_{2}} & \ldots & \frac{\partial g_{2}(x ; \theta)}{\partial x_{n}} \\
\vdots & \vdots & \ddots & \vdots \\
\frac{\partial g_{n}(x ; \theta)}{\partial x_{1}} & \frac{\partial g_{n}(x ; \theta)}{\partial x_{2}} & \ldots & \frac{\partial g_{n}(x ; \theta)}{\partial x_{n}}
\end{array}\right) .
$$

The Jacobian matrix is required to be invertible almost everywhere, which justifies local invertibility of function $g$ by the implicit function theory. The conditions for justifying unbiasedness are referred to Peng et al. (2018) and Peng et al. (2021). Let $e_{i}$ be the $i$-th unit vector. We define

$$
\begin{aligned}
d(x ; \theta):= & \sum_{i=1}^{n} e_{i}^{T} J_{g}^{-1}(x ; \theta)\left(\partial_{x_{i}} J_{g}(x ; \theta)\right) J_{g}^{-1}(x ; \theta) \partial_{\theta} g(x ; \theta) \\
& -\operatorname{trace}\left(J_{g}^{-1}(x ; \theta) \partial_{\theta} J_{g}(x ; \theta)\right)-\left(J_{g}^{-1}(x ; \theta) \partial_{\theta} g(x ; \theta)\right)^{T} \nabla_{x} \log f_{X}(x ; \theta),
\end{aligned}
$$

where and $\partial_{y} M(y)$ is the matrix obtained by differentiating $M$ with respect to $y$ element-wise. From Peng et al. (2018), when the tails of $f_{X}(\cdot ; \theta)$ go smoothly to zero fast enough, then, under certain regularity conditions, it can be shown that an unbiased GLR estimator for (3) is given by

$$
G(X ; \theta):=\varphi(g(X ; \theta)) w(X ; \theta) \quad \text { with } \quad w(x ; \theta):=\frac{\partial \log f_{X}(x ; \theta)}{\partial \theta}+d(x ; \theta) .
$$

As a particular stochastic activity network (SAN) example, the output could be the maximum of the durations of activities on different paths. By the GLR method under general framework (4), we can consider quantile sensitivity for $\max _{i=1, \ldots, n} g_{i}(X ; \theta)$, which has a distribution function expressed as

$$
\mathbb{E}\left[\mathbf{1}\left\{\max _{i=1, \ldots, n} g_{i}(X ; \boldsymbol{\theta}) \leq y\right\}\right]=\mathbb{E}\left[\prod_{i=1}^{n} \mathbf{1}\left\{g_{i}(X ; \boldsymbol{\theta})-y \leq 0\right\}\right],
$$

with $\varphi(y)=\prod_{i=1}^{n} \mathbf{1}\left\{y_{i} \leq 0\right\}$. Peng et al. (2021) consider the case when $X$ is a vector of uniform random numbers $U=\left(U_{1}, \ldots, U_{n}\right)$ such that $\Omega=(0,1)^{n}$ and $\partial \log f_{X}(x ; \theta) / \partial \theta=0$. Then under certain regularity conditions, we have the following unbiased GLR estimator for (3):

$$
\widetilde{G}(U ; \theta):=\sum_{i=1}^{n}\left[\varphi\left(g\left(\bar{U}_{i} ; \theta\right)\right) r_{i}\left(\bar{U}_{i} ; \theta\right)-\varphi\left(g\left(\underline{U}_{i} ; \theta\right)\right) r_{i}\left(\underline{U}_{i} ; \theta\right)\right]+\varphi(g(U ; \theta)) d(U ; \theta),
$$

where

$$
r_{i}(x ; \theta):=\left(J_{g}^{-1}(x ; \theta) \partial_{\theta} g(x ; \theta)\right)^{T} e_{i}, \quad i=1, \ldots, n .
$$

\section{VARIANCE REDUCTION}

CMC methods can reduce the variance and smooth the performance function in simulation by conditioning on certain events or random variables and then integrating out the remaining randomness (Asmussen and Glynn (2007)). For an estimator $H(Z)$, we have

$$
\mathbb{E}[H(Z)]=\mathbb{E}[\widehat{H}(\widehat{Z})]
$$


where $\widehat{H}(\widehat{Z}):=\mathbb{E}[H(Z) \mid \widehat{Z}]$, with $\widehat{Z}$ being a subset of input random variables in $Z$. The variance reduction for the conditional estimator $\widehat{H}(\widehat{Z})$ can be seen from the following variance decomposition formula:

$$
\begin{aligned}
\operatorname{Var}(H(Z)) & =\operatorname{Var}(\mathbb{E}[H(Z) \mid \widehat{Z}])+\mathbb{E}[\operatorname{Var}(H(Z) \mid \widehat{Z})] \\
& \geq \operatorname{Var}(\widehat{H}(\widehat{Z})) .
\end{aligned}
$$

Typically, $\widehat{H}(\widehat{Z})$ is smoother than $H(Z)$, due to the integration taken in the conditional expectation.

Quasi-Monte Carlo (QMC) refers to a class of deterministic numerical integration methods in which the integrand is evaluated at a fixed set of $m$ points, and the average is used as an approximation. One limitation of the method is that it is very hard to estimate the approximation error in practice. RQMC takes the QMC points and randomizes them in a way that each point has the uniform distribution over $(0,1)^{n}$, so that each randomized point represents a proper realization of $U$ while the set of $m$ points still covers the unit hypercube $(0,1)^{n}$ more uniformly than typical independent random points (so the points are not independent) (L'Ecuyer 2018).

In general, for a given function $p$, RQMC estimates the integral $\mu=\int_{(0,1)^{n}} p(u) d u$ by the average

$$
\hat{\mu}_{m}:=\frac{1}{m} \sum_{j=1}^{m} p\left(U^{(j)}\right)
$$

where $U^{(1)}, \ldots, U^{(m)}$ form an RQMC point set. The most common types of QMC point set constructions are lattice rules, polynomial lattices rules, and digital nets (Dick and Pillichshammer 2010; L'Ecuyer 2009). For lattice rules, an appropriate randomization is a random shift modulo 1, which adds a single uniform random point to all the lattice points, and retains the shifted points that are in $(0,1)^{n}$ as the $m$ RQMC points. This randomization preserves the lattice structure, and there are explicit expressions for $\operatorname{Var}\left[\hat{\mu}_{m}\right]$ in terms of the Fourier coefficients of $h$, and computable bounds on this variance for certain classes of smooth functions (L'Ecuyer and Lemieux 2000; L'Ecuyer and Munger 2012; L'Ecuyer and Munger 2016). When the mixed derivatives of $p$ are sufficiently smooth, the variance can converge at a faster rate than $\mathscr{O}\left(\mathrm{m}^{-1}\right)$, sometimes nearly $\mathscr{O}\left(\mathrm{m}^{-2}\right)$ and even faster in some cases. When $p$ is not smooth (e.g., discontinuous), these convergence rate results do not apply, although weaker results do apply (He and Wang 2015), and even when the convergence rate is not improved, the variance is often reduced by a constant factor. For polynomial lattices rules and digital nets in general, which include Sobol' points, the random shift does not preserve the structure and net properties, but other appropriate randomizations do, including nested uniform scrambling, some affine scrambles, and random digital shifts. Variance bounds and convergence rate results are also available for these rules (Dick and Pillichshammer 2010; L'Ecuyer 2009).

Our model formulation (5) in terms of a function of independent $\mathscr{U}(0,1)$ random variables makes it an obvious candidate for the application of RQMC, which is designed exactly for this type of formulation. For formulation (4), suppose $X$ can be generated by $\Gamma(U)$, and RQMC can be applied. For example, when $X_{1}, \ldots, X_{n}$ are independent random variables with marginal distribution functions $F_{1}, \ldots, F_{n}$, then they can be generated by $X_{i}=F_{i}^{-1}\left(U_{i}\right), i=1, \ldots, n$. For the $\alpha$-quantile sensitivity estimation, the cdf $F(\cdot ; \theta)$ of $p(U)$ can be estimated by its empirical RQMC counterpart

$$
\mathscr{F}_{m}(y):=\frac{1}{m} \sum_{j=1}^{m} \mathbf{1}\left\{p\left(U^{(j)}\right) \leq y\right\}
$$

where $\left\{U^{(1)}, \ldots, U^{(m)}\right\}$ is an RQMC point set, and the quantile $q_{\alpha}(\theta)$ can be estimated by the pseudo-inverse $\mathscr{F}_{m}^{-1}(\alpha)$. We can also use RQMC points in the sensitivity estimate (2).

\section{NUMERICAL EXPERIMENTS}

We apply the proposed method to two applications. GLR is compared with the finite difference method using common random numbers $(\operatorname{FDC}(\delta))$, where $\delta$ is the perturbation size. The GLR method together 
with CMC is called conditional GLR (CGLR), and CGLR together with RQMC is denoted as CGLRQ. For RQMC, we use the Sobol sequence scrambled by the algorithm of Matousek (1998) in Matlab. In the two examples, the sample size for estimating quantile sensitivities are set as $m=2^{15}$ for the standard Monte Carlo and RQMC estimators, and the quantile senstivities and standard errors of the estimators are estimated by $10^{4}$ independent experiments.

\subsection{TOY EXAMPLE}

To illustrate, we estimate quantile sensitivity of a simple stochastic model $\theta X_{1}+X_{2}+U$, where $X_{1}, X_{2}$ are standard normal random variables with the common $\operatorname{cdf} \Phi(\cdot)$, and $U$ is an independent uniform random variable $\mathscr{U}(0,1)$. In the stochastic model for deriving GLR estimators, the input random variable could either be taken as the normal random variables $X_{1}, X_{2}$ or uniform random number $U$. If we choose $U$ to be the input random variable, then $h\left(u ; \theta, x_{1}, x_{2}\right)=\theta x_{1}+x_{2}+u, \partial h\left(u ; \theta, x_{1}, x_{2}\right) / \partial u=1, \partial h\left(u ; \theta, x_{1}, x_{2}\right) / \partial \theta=x_{1}$, and other derivatives in (5) are zeros, so GLR estimators for distribution sensitivities are given by

$$
\begin{aligned}
& \widetilde{G}_{1,1}\left(U ; \theta, X_{1}, X_{2}\right)=\mathbf{1}\left\{\theta X_{1}+X_{2}+1 \leq y\right\} X_{1}-\mathbf{1}\left\{\theta X_{1}+X_{2} \leq y\right\} X_{1} \\
& \widetilde{G}_{2,1}\left(U ; \theta, X_{1}, X_{2}\right)=\mathbf{1}\left\{\theta X_{1}+X_{2} \leq y\right\}-\mathbf{1}\left\{\theta X_{1}+X_{2}+1 \leq y\right\}
\end{aligned}
$$

By conditioning on $X_{1}$, we have

$$
\begin{aligned}
\mathbb{E}\left[\widetilde{G}_{1,1}\left(U ; y, \theta, X_{1}, X_{2}\right)\right] & =\mathbb{E}\left[\mathbb{E}\left[\mathbf{1}\left\{\theta X_{1}+X_{2}+1 \leq y\right\} X_{1}-\mathbf{1}\left\{\theta X_{1}+X_{2} \leq y\right\} X_{1} \mid X_{1}\right]\right] \\
& =\mathbb{E}\left[\left(\Phi\left(y-\theta X_{1}-1\right)-\Phi\left(y-\theta X_{1}\right)\right) X_{1}\right], \\
\mathbb{E}\left[\widetilde{G}_{2,1}\left(U ; y, \theta, X_{1}, X_{2}\right)\right] & =\mathbb{E}\left[\mathbb{E}\left[\mathbf{1}\left\{\theta X_{1}+X_{2} \leq y\right\}-\mathbf{1}\left\{\theta X_{1}+X_{2}+1 \leq y\right\} \mid X_{1}\right]\right] \\
& =\mathbb{E}\left[\Phi\left(y-\theta X_{1}\right)-\Phi\left(y-\theta X_{1}-1\right)\right] .
\end{aligned}
$$

We can take the expressions inside the expectations on the right-hand sides of the above equations as conditional GLR estimators. From this derivation, three quantile sensitivity estimators of the form (2) using GLR, CGLR, and CGLRQ to estimate distribution sensitivities are referred to as GLR-1, CGLR-1, and CGLRQ-1, respectively.

If we choose $X_{1}$ to be the input random variable, then $h\left(x_{1}, x_{2} ; \theta, u\right)=\theta x_{1}+x_{2}+u, \partial h\left(x_{1}, x_{2} ; \theta, u\right) / \partial x_{1}=$ $\theta, \partial h\left(x_{1}, x_{2} ; \theta, u\right) / \partial \theta=x_{1}, \partial^{2} h\left(x_{1}, x_{2} ; \theta, u\right) / \partial \theta \partial x_{1}=1, \nabla_{x} \log f_{X}\left(x_{1}, x_{2}\right)=-\left(x_{1}, x_{2}\right)$, and the other derivatives in (4) are zeros, so GLR estimators for distribution sensitivities can be given by

$$
\begin{aligned}
& G_{1,1}\left(X_{1}, X_{2} ; y, \theta, U\right)=\frac{1}{\theta} \mathbf{1}\left\{\theta X_{1}+X_{2}+U \leq y\right\}\left(X_{1}^{2}-1\right), \\
& G_{2,1}\left(X_{1}, X_{2} ; y, \theta, U\right)=-\frac{1}{\theta} \mathbf{1}\left\{\theta X_{1}+X_{2}+U \leq y\right\} X_{1}
\end{aligned}
$$

By conditioning on $X_{1}, U$, we have

$$
\begin{aligned}
& \mathbb{E}\left[G_{1,1}\left(X_{1}, X_{2} ; y, \theta, U\right)\right]=\mathbb{E}\left[\mathbb{E}\left[\frac{1}{\theta} \mathbf{1}\left\{\theta X_{1}+X_{2}+U \leq y\right\} X_{1}^{2} \mid X_{1}, U\right]\right]=\frac{1}{\theta} \mathbb{E}\left[\Phi\left(y-\theta X_{1}-U\right) X_{1}^{2}\right], \\
& \mathbb{E}\left[G_{2,1}\left(X_{1}, X_{2} ; y, \theta, U\right)\right]=\mathbb{E}\left[\mathbb{E}\left[-\frac{1}{\theta} \mathbf{1}\left\{\theta X_{1}+X_{2}+U \leq y\right\} X_{1} \mid X_{1}, U\right]\right]=-\frac{1}{\theta} \mathbb{E}\left[\Phi\left(y-\theta X_{1}-U\right) X_{1}\right] .
\end{aligned}
$$

We can take the expressions inside the expectations on the right-hand sides of the above equations as conditional GLR estimators. From this derivation, three quantile sensitivity estimators of the form (2) using 
Peng, Fu, Hu, L'Ecuyer, and Tuffin

Table 1:

\begin{tabular}{|c|c|c|c|c|c|}
\hline & $\alpha=0.1$ & $\alpha=0.3$ & $\alpha=0.5$ & $\alpha=0.7$ & $\alpha=0.9$ \\
\hline GLR-1 & $-0.888 \pm 1.4 \times 10^{-4}$ & $-0.363 \pm 1.0 \times 10^{-4}$ & $0 \pm 1.0 \times 10^{-4}$ & $0.363 \pm 1.0 \times 10^{-4}$ & $0.887 \pm 1.4 \times 10^{-4}$ \\
CGLR-1 & $-0.888 \pm 1.0 \times 10^{-4}$ & $-0.363 \pm 8 \times 10^{-5}$ & $0 \pm 7 \times 10^{-5}$ & $0.364 \pm 8 \times 10^{-5}$ & $0.888 \pm 1.0 \times 10^{-4}$ \\
CGLRQ-1 & $-0.888 \pm 4.7 \times 10^{-5}$ & $-0.363 \pm 4.1 \times 10^{-5}$ & $0 \pm 3.9 \times 10^{-5}$ & $0.363 \pm 4.1 \times 10^{-5}$ & $0.888 \pm 4.7 \times 10^{-5}$ \\
GLR-2 & $-0.888 \pm 2.3 \times 10^{-4}$ & $-0.363 \pm 1.8 \times 10^{-4}$ & $0 \pm 2.1 \times 10^{-4}$ & $0.363 \pm 2.9 \times 10^{-4}$ & $0.890 \pm 7.3 \times 10^{-4}$ \\
CGLR-2 & $-0.888 \pm 2.0 \times 10^{-4}$ & $-0.363 \pm 1.8 \times 10^{-4}$ & $0 \pm 2.0 \times 10^{-4}$ & $0.364 \pm 2.8 \times 10^{-4}$ & $0.889 \pm 7.2 \times 10^{-4}$ \\
CGLRQ-2 & $-0.888 \pm 4.9 \times 10^{-5}$ & $-0.363 \pm 4.1 \times 10^{-5}$ & $0 \pm 3.9 \times 10^{-5}$ & $0.363 \pm 4.1 \times 10^{-5}$ & $0.888 \pm 4.9 \times 10^{-5}$ \\
FDC(0.01) & $-0.888 \pm 1.2 \times 10^{-3}$ & $-0.364 \pm 8.5 \times 10^{-4}$ & $0 \pm 7.9 \times 10^{-4}$ & $0.365 \pm 8.6 \times 10^{-4}$ & $0.890 \pm 1.2 \times 10^{-3}$ \\
FDC(0.1) & $-0.911 \pm 3.9 \times 10^{-4}$ & $-0.373 \pm 2.7 \times 10^{-4}$ & $0 \pm 2.6 \times 10^{-4}$ & $0.372 \pm 2.8 \times 10^{-4}$ & $0.910 \pm 3.9 \times 10^{-4}$ \\
\hline
\end{tabular}

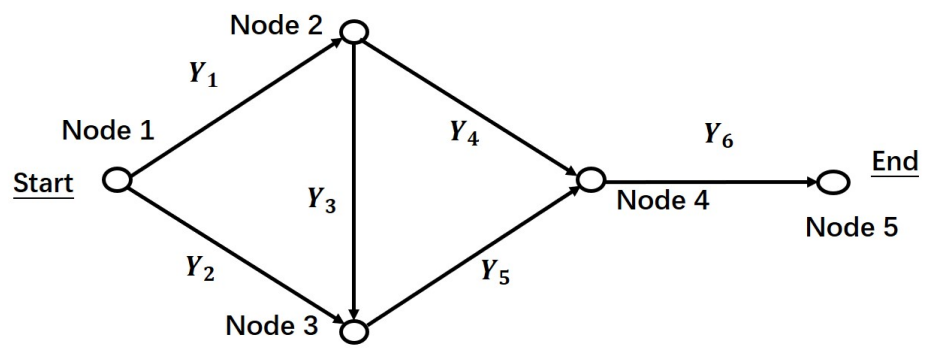

Figure 1: A SAN with seven activities.

GLR, CGLR, and CGLRQ to estimate distribution sensitivities are referred to as GLR-2, CGLR-2, and CGLRQ-2, respectively.

For our experiments, we took $\theta=1$ and five probability levels: $\alpha=0.1, \alpha=0.3, \alpha=0.5, \alpha=0.7$, and $\alpha=0.9$. From Table 1, we can see that the quantile sensitivity estimates by GLR-1, CGLR-1, CGLRQ-1, GLR-2, CGLR-2, CGLRQ-2, and FDC(0.01) are statistically indistinguishable, whereas the quantile sensitivity estimates by $\mathrm{FDC}(0.1)$ lie outside of the $90 \%$ confidence intervals of other methods. GLR leads to smaller variance than FDC, the variances of GLR-1, CGLR-1, CGLRQ-1 are smaller than GLR-2, CGLR-2, CGLRQ-2, respectively, and the CMC and RQMC lead to significant variance reduction.

\subsection{STOCHASTIC ACTIVITY NETWORK (SAN)}

We estimate quantile sensitivity for a simple SAN. There are three different paths representing the tasks to reach the final stage of a project, i.e., $(1,4,6),(2,5,6), \quad(1,3,5,6)$. The completion time for the entire project is $\max \left(Y_{1}+Y_{4}+Y_{6}, Y_{2}+Y_{5}+Y_{6}, Y_{1}+Y_{3}+Y_{5}+Y_{6}\right)$, and the sample performance for the cdf of the completion time is

$$
\mathbf{1}\left\{\max \left(Y_{1}+Y_{4}+Y_{6}, Y_{2}+Y_{5}+Y_{6}, Y_{1}+Y_{3}+Y_{5}+Y_{6}\right) \leq y\right\} .
$$

We assume that the first three activities follow independent exponential distributions: $Y_{i}=-\frac{1}{\lambda_{i}} \log \left(U_{i}\right)$, $i=1,2,3$, and the other three activities follow independent log-normal distributions, $Y_{i}=\exp \left(\mu_{i}+\sigma_{i} X_{i}\right)$, $i=4,5,6$, where $X_{i}$ 's are standard normal random variables. Let $\lambda_{1}=\theta$. The distribution function $F(y ; \theta)$ of the completion time $Y$ is

$$
F(y ; \theta)=\mathbb{E}\left[\mathbf{1}\left\{Y_{1}+\max \left(Y_{4}, Y_{3}+Y_{5}\right)+Y_{6}-y \leq 0\right\} \mathbf{1}\left\{Y_{2}+Y_{5}+Y_{6}-y \leq 0\right\}\right]
$$

If we keep the original indicator above, then the image of $g$ is in $\mathbb{R}^{3}$ and we need three inputs. With the rewriting, the image of $g$ is in $\mathbb{R}^{2}$ and we select two inputs in a way that the Jacobian is invertible. To estimate the two distribution sensitivities, we can view $\left(U_{1}, U_{2}\right)$ as the input random variables in the 
Peng, Fu, Hu, L'Ecuyer, and Tuffin

stochastic model of Peng et al. (2021), and we have

$$
\begin{aligned}
& g_{1}\left(U_{1}, U_{2} ; \theta, y, U_{3}, X_{4}, X_{5}, X_{6}\right)=-\frac{1}{\lambda_{1}} \log U_{1}+\max \left(Y_{4}, Y_{3}+Y_{5}\right)+Y_{6}-z, \\
& g_{2}\left(U_{1}, U_{2} ; \theta, y, U_{3}, X_{4}, X_{5}, X_{6}\right)=-\frac{1}{\lambda_{2}} \log U_{2}+Y_{5}+Y_{6}-z, \\
& \partial_{\theta} g\left(u_{1}, u_{2} ; \theta, y, u_{3}, x_{4}, x_{5}, x_{6}\right)=\left(\log u_{1} / \lambda_{1}^{2}, 0\right)^{T}, \quad \partial_{y} g\left(u_{1}, u_{2} ; \theta, y, u_{3}, x_{4}, x_{5}, x_{6}\right)=-(1,1)^{T} .
\end{aligned}
$$

The Jacobian matrix and its inverse are

$$
\begin{gathered}
J_{g}\left(u_{1}, u_{2} ; \theta, y\right)=-\left(\begin{array}{cc}
\frac{1}{\lambda_{1} u_{1}} & 0 \\
0 & \frac{1}{\lambda_{2} u_{2}}
\end{array}\right), \quad J_{g}^{-1}\left(u_{1}, u_{2} ; \theta, y\right)=-\left(\begin{array}{cc}
\lambda_{1} u_{1} & 0 \\
0 & \lambda_{2} u_{2}
\end{array}\right), \\
\partial_{u_{1}} J_{g}\left(u_{1}, u_{2} ; \theta, y\right)=\left(\begin{array}{cc}
\frac{1}{\lambda_{1} u_{1}^{2}} & 0 \\
0 & 0
\end{array}\right), \partial_{u_{2}} J_{g}\left(u_{1}, u_{2} ; \theta, y\right)=\left(\begin{array}{cc}
0 & 0 \\
0 & \frac{1}{\lambda_{2} u_{2}^{2}}
\end{array}\right), \partial_{\theta} J_{g}\left(u_{1}, u_{2} ; \theta, y\right)=\left(\begin{array}{cc}
\frac{1}{\lambda_{1}^{2} u_{1}} & 0 \\
0 & 0
\end{array}\right)
\end{gathered}
$$

and

$$
\begin{aligned}
& r_{1}\left(u_{1}, u_{2} ; \theta\right)=\frac{1}{\lambda_{1}} u_{1} \log u_{1}, \quad r_{2}\left(u_{1}, u_{2} ; \theta\right)=0, \quad d\left(u_{1}, u_{2} ; \theta\right)=\frac{1}{\lambda_{1}}\left(\log u_{1}+1\right), \\
& \tilde{r}_{1}\left(u_{1}, u_{2} ; \theta\right)=\lambda_{1} u_{1}, \quad \tilde{r}_{2}\left(u_{1}, u_{2} ; \theta\right)=\lambda_{2} u_{2}, \quad \tilde{d}\left(u_{1}, u_{2} ; \theta\right)=-\lambda_{1}-\lambda_{2} .
\end{aligned}
$$

Then the GLR estimator of $\partial F(y ; \theta) / \partial \theta$ is given by

$$
G_{1}\left(U_{1}, U_{2} ; \theta, y, U_{3}, X_{4}, X_{5}, X_{6}\right)=\left(\frac{1}{\lambda_{1}}-Y_{1}\right) \mathbf{1}\left\{\max \left(Y_{1}+Y_{4}+Y_{6}, Y_{2}+Y_{5}+Y_{6}, Y_{1}+Y_{3}+Y_{5}+Y_{6}\right) \leq y\right\},
$$

and the GLR estimator of $\partial F(y ; \theta) / \partial y$ is given by

$$
\begin{aligned}
G_{2}\left(U_{1}, U_{2} ; \theta, y, U_{3}, X_{4}, X_{5}, X_{6}\right) & =\lambda_{1} \mathbf{1}\left\{\max \left(Y_{4}+Y_{6}, Y_{2}+Y_{5}+Y_{6}, Y_{3}+Y_{5}+Y_{6}\right) \leq y\right\} \\
& +\lambda_{2} \mathbf{1}\left\{\max \left(Y_{1}+Y_{4}+Y_{6}, Y_{5}+Y_{6}, Y_{3}+Y_{5}+Y_{6}\right) \leq y\right\} \\
& -\left(\lambda_{1}+\lambda_{2}\right) \mathbf{1}\left\{\max \left(Y_{1}+Y_{4}+Y_{6}, Y_{2}+Y_{5}+Y_{6}, Y_{1}+Y_{3}+Y_{5}+Y_{6}\right) \leq y\right\} .
\end{aligned}
$$

Furthermore, we have

$$
\begin{aligned}
& \mathbb{E}\left[G_{1}\left(U_{1}, U_{2} ; \theta, y, U_{3}, X_{4}, X_{5}, X_{6}\right)\right]=\mathbb{E}\left[\mathbb{E}\left[G_{1}\left(U_{1}, U_{2} ; \theta, y, U_{3}, X_{4}, X_{5}, X_{6}\right) \mid Y_{1}, \ldots, Y_{5}\right]\right] \\
= & \mathbb{E}\left[\left(\frac{1}{\lambda_{1}}-Y_{1}\right) \Phi\left(\frac{1}{\sigma_{6}}\left(\log \left[(y-\widetilde{Y})^{+}\right]-\mu_{6}\right)\right)\right],
\end{aligned}
$$

and

$$
\begin{aligned}
& \mathbb{E}\left[G_{2}\left(U_{1}, U_{2} ; \theta, y, U_{3}, X_{4}, X_{5}, X_{6}\right)\right]=\mathbb{E}\left[\mathbb{E}\left[G_{2}\left(U_{1}, U_{2} ; \theta, y, U_{3}, X_{4}, X_{5}, X_{6}\right) \mid Y_{1}, \ldots, Y_{5}\right]\right] \\
= & \lambda_{1} \mathbb{E}\left[\Phi\left(\frac{1}{\sigma_{6}}\left(\log \left[\left(y-\max \left(Y_{4}, Y_{2}+Y_{5}, Y_{3}+Y_{5}\right)\right)^{+}\right]-\mu_{6}\right)\right)\right] \\
& +\lambda_{2} \mathbb{E}\left[\Phi\left(\frac{1}{\sigma_{6}}\left(\log \left[\left(y-\max \left(Y_{1}+Y_{4}, Y_{5}, Y_{1}+Y_{3}+Y_{5}\right)\right)^{+}\right]-\mu_{6}\right)\right)\right] \\
& -\left(\lambda_{1}+\lambda_{2}\right) \mathbb{E}\left[\Phi\left(\frac{1}{\sigma_{6}}\left(\log \left[(y-\tilde{Y})^{+}\right]-\mu_{6}\right)\right)\right],
\end{aligned}
$$


where $\widetilde{Y}=\max \left(Y_{1}+Y_{4}, Y_{2}+Y_{5}, Y_{1}+Y_{3}+Y_{5}\right)$. We can take the expressions inside the expectations on the right-hand sides of the above equation as conditional GLR estimators. From this derivation, the three quantile sensitivity estimators of the form (2) using GLR, CGLR, and CGLRQ to estimate distribution sensitivities are referred to as GLR-1, CGLR-1, and CGLRQ-1, respectively.

On the other hand, we have

$$
F(y ; \theta)=\mathbb{E}\left[\mathbf{1}\left\{Y_{4}+Y_{1}+Y_{6}-y \leq 0\right\} \mathbf{1}\left\{Y_{5}+\max \left(Y_{2}, Y_{1}+Y_{3}\right)+Y_{6}-y \leq 0\right\}\right],
$$

and we can also let $\left(X_{4}, X_{5}\right)$ be the input random variables in the stochastic model of Peng et al. (2018), leading to

$$
\begin{aligned}
& g_{1}\left(X_{4}, X_{5} ; \theta, y, U_{1}, U_{2}, U_{3}, X_{6}\right)=\exp \left(\mu_{4}+\sigma_{4} X_{4}\right)+Y_{1}+Y_{6}-y, \\
& g_{2}\left(X_{4}, X_{5} ; \theta, y, U_{1}, U_{2}, U_{3}, X_{6}\right)=\exp \left(\mu_{5}+\sigma_{5} X_{5}\right)+\max \left(Y_{2}, Y_{1}+Y_{3}\right)+Y_{6}-y, \\
& \partial_{\theta} g\left(x_{4}, x_{5} ; \theta, y, u_{1}, u_{2}, u_{3}, x_{6}\right)=\log u_{1} / \lambda_{1}^{2}\left(1, \mathbf{1}\left\{\log u_{1} / \lambda_{1}+\log u_{3} / \lambda_{3}<\log u_{2} / \lambda_{2}\right\}\right)^{T}, \\
& \partial_{y} g\left(x_{4}, x_{5} ; \theta, y, u_{1}, u_{2}, u_{3}, x_{6}\right)=-(1,1)^{T}, \\
& \nabla \log f_{\left(X_{4}, X_{5}\right)}\left(x_{4}, x_{5}\right)=-\left(x_{4}, x_{5}\right)^{T} .
\end{aligned}
$$

The Jacobian matrix and its inverse are

$$
\begin{gathered}
J_{g}\left(x_{4}, x_{5}\right)=\left(\begin{array}{cc}
\sigma_{4} e^{\mu_{4}+\sigma_{4} x_{4}} & 0 \\
0 & \sigma_{5} e^{\mu_{5}+\sigma_{5} x_{5}}
\end{array}\right), \quad J_{g}^{-1}\left(x_{4}, x_{5}\right)=\left(\begin{array}{cc}
\frac{1}{\sigma_{4}} e^{-\mu_{4}-\sigma_{4} x_{4}} & 0 \\
0 & \frac{1}{\sigma_{5}} e^{-\mu_{5}-\sigma_{5} x_{5}}
\end{array}\right), \\
\partial_{x_{4}} J_{g}\left(x_{4}, x_{5}\right)=\left(\begin{array}{cc}
\sigma_{4}^{2} e^{\mu_{4}+\sigma_{4} x_{4}} & 0 \\
0 & 0
\end{array}\right), \quad \partial_{x_{5}} J_{g}\left(x_{4}, x_{5}\right)=\left(\begin{array}{cc}
0 & 0 \\
0 & \sigma_{5}^{2} e^{\mu_{5}+\sigma_{5} x_{5}}
\end{array}\right),
\end{gathered}
$$

and

$$
\begin{aligned}
& d\left(x_{4}, x_{5} ; \theta\right)=\frac{\log u_{1}}{\lambda_{1}^{2}}\left[\left(1+\frac{x_{4}}{\sigma_{4}}\right) e^{-\mu_{4}-\sigma_{4} x_{4}}+\mathbf{1}\left\{\frac{\log u_{1}}{\lambda_{1}}+\frac{\log u_{3}}{\lambda_{3}}<\frac{\log u_{2}}{\lambda_{2}}\right\}\left(1+\frac{x_{5}}{\sigma_{5}}\right) e^{-\mu_{5}-\sigma_{5} x_{5}}\right], \\
& \tilde{d}\left(x_{4}, x_{5} ; \theta\right)=-\left(1+\frac{x_{4}}{\sigma_{4}}\right) e^{-\mu_{4}-\sigma_{4} x_{4}}-\left(1+\frac{x_{5}}{\sigma_{5}}\right) e^{-\mu_{5}-\sigma_{5} x_{5}} .
\end{aligned}
$$

Then the GLR estimator of $\partial F(y ; \theta) / \partial \theta$ is given by

$$
G_{1}\left(X_{4}, X_{5} ; \theta, y, U_{1}, U_{2}, U_{3}, X_{6}\right)=-\mathbf{1}\{Y \leq z\} \frac{Y_{1}}{\lambda_{1}}\left[\left(1+\frac{X_{4}}{\sigma_{4}}\right) \frac{1}{Y_{4}}+\mathbf{1}\left\{Y_{1}+Y_{3}>Y_{2}\right\}\left(1+\frac{X_{5}}{\sigma_{5}}\right) \frac{1}{Y_{5}}\right] .
$$

the GLR estimator of $\partial F(y ; \theta) / \partial y$ is given by

$$
G_{2}\left(X_{4}, X_{5} ; \theta, y, U_{1}, U_{2}, U_{3}, X_{6}\right)=-\mathbf{1}\{Y \leq z\}\left[\left(1+\frac{X_{4}}{\sigma_{4}}\right) \frac{1}{Y_{4}}+\left(1+\frac{X_{5}}{\sigma_{5}}\right) \frac{1}{Y_{5}}\right] .
$$

Furthermore, we have

$$
\begin{aligned}
& \mathbb{E}\left[G_{1}\left(X_{4}, X_{5} ; \theta, y, U_{1}, U_{2}, U_{3}, X_{6}\right)\right]=\mathbb{E}\left[\mathbb{E}\left[G_{1}\left(X_{4}, X_{5} ; \theta, y, U_{1}, U_{2}, U_{3}, X_{6}\right) \mid Y_{1}, \ldots, Y_{5}\right]\right] \\
= & -\mathbb{E}\left[\frac{Y_{1}}{\lambda_{1}}\left[\left(1+\frac{X_{4}}{\sigma_{4}}\right) \frac{1}{Y_{4}}+\mathbf{1}\left\{Y_{1}+Y_{3}>Y_{2}\right\}\left(1+\frac{X_{5}}{\sigma_{5}}\right) \frac{1}{Y_{5}}\right] \Phi\left(\frac{1}{\sigma_{6}}\left(\log \left[(y-\widetilde{Y})^{+}\right]-\mu_{6}\right)\right)\right] .
\end{aligned}
$$


Peng, Fu, Hu, L'Ecuyer, and Tuffin

Table 2:

\begin{tabular}{|c|c|c|c|c|c|}
\hline & $\alpha=0.1$ & $\alpha=0.3$ & $\alpha=0.5$ & $\alpha=0.7$ & $\alpha=0.9$ \\
\hline GLR-1 & $-0.456 \pm 1.0 \times 10^{-4}$ & $-0.697 \pm 1.3 \times 10^{-4}$ & $-0.907 \pm 2.0 \times 10^{-4}$ & $-1.136 \pm 3.9 \times 10^{-4}$ & $-1.374 \pm 1.6 \times 10^{-3}$ \\
CGLR-1 & $-0.456 \pm 6.8 \times 10^{-5}$ & $-0.696 \pm 1.0 \times 10^{-4}$ & $-0.907 \pm 1.7 \times 10^{-4}$ & $-1.136 \pm 3.6 \times 10^{-4}$ & $-1.374 \pm 1.5 \times 10^{-3}$ \\
CGLRQ-1 & $-0.456 \pm 5.0 \times 10^{-5}$ & $-0.696 \pm 5.6 \times 10^{-5}$ & $-0.907 \pm 6.8 \times 10^{-5}$ & $-1.135 \pm 1.1 \times 10^{-4}$ & $-1.377 \pm 3.1 \times 10^{-4}$ \\
GLR-2 & $-0.456 \pm 1.0 \times 10^{-4}$ & $-0.700 \pm 6.7 \times 10^{-4}$ & $-0.911 \pm 1.1 \times 10^{-3}$ & $-1.163 \pm 2.9 \times 10^{-3}$ & $-0.094 \pm 7.4 \times 10^{-1}$ \\
CGLR-2 & $-0.456 \pm 3.0 \times 10^{-4}$ & $-0.698 \pm 5.4 \times 10^{-4}$ & $-0.913 \pm 1.0 \times 10^{-3}$ & $-1.161 \pm 2.5 \times 10^{-3}$ & $-1.026 \pm 2.5 \times 10^{-1}$ \\
CGLRQ-2 & $-0.456 \pm 2.6 \times 10^{-4}$ & $-0.697 \pm 4.4 \times 10^{-4}$ & $-0.910 \pm 7.9 \times 10^{-4}$ & $-1.145 \pm 1.7 \times 10^{-3}$ & $-1.957 \pm 2.0 \times 10^{-1}$ \\
FDC(0.01) & $-0.453 \pm 9.5 \times 10^{-4}$ & $-0.690 \pm 9.8 \times 10^{-4}$ & $-0.897 \pm 1.2 \times 10^{-3}$ & $-1.123 \pm 1.6 \times 10^{-3}$ & $-1.366 \pm 3.3 \times 10^{-3}$ \\
FDC(0.1) & $-0.423 \pm 2.9 \times 10^{-4}$ & $-0.639 \pm 3.0 \times 10^{-4}$ & $-0.826 \pm 3.6 \times 10^{-4}$ & $-1.024 \pm 5.0 \times 10^{-4}$ & $-1.223 \pm 1.0 \times 10^{-3}$ \\
\hline
\end{tabular}

and

$$
\begin{aligned}
& \mathbb{E}\left[G_{2}\left(X_{4}, X_{5} ; \theta, y, U_{1}, U_{2}, U_{3}, X_{6}\right)\right]=\mathbb{E}\left[\mathbb{E}\left[G_{2}\left(X_{4}, X_{5} ; \theta, y, U_{1}, U_{2}, U_{3}, X_{6}\right) \mid Y_{1}, \ldots, Y_{5}\right]\right] \\
= & -\mathbb{E}\left[\left(\frac{1}{Y_{4}}+\frac{X_{4}}{\sigma_{4} Y_{4}}+\frac{1}{Y_{5}}+\frac{X_{5}}{\sigma_{5} Y_{5}}\right) \Phi\left(\frac{1}{\sigma_{6}}\left(\log \left[(y-\widetilde{Y})^{+}\right]-\mu_{6}\right)\right)\right] .
\end{aligned}
$$

We can take the expressions inside the expectations on the right-hand sides of the above equations as conditional GLR estimators. From this derivation, the three quantile sensitivity estimators of the form (2) using GLR, CGLR, and CGLRQ to estimate distribution sensitivities are referred to as GLR-2, CGLR-2, and CGLRQ-2, respectively.

In the experiment, we set $\lambda_{i}=1, \mu_{i}=0, \sigma_{i}=1, i=1,2,3$, and test five probability levels at $\alpha=0.1$, $\alpha=0.3, \alpha=0.5, \alpha=0.7$, and $\alpha=0.9$. From Table 2 , we can see that the quantile sensitivity estimates by GLR-1, CGLR-1, CGLRQ-1, GLR-2, CGLR-2, CGLRQ-2 are statistically indistinguishable, FDC(0.01) appears to be slightly biased, and FDC(0.1) exhibits a significant bias compared to the GLR method. The variances of GLR-1, CGLR-1, CGLRQ-1 are smaller than GLR-2, CGLR-2, CGLRQ-2, respectively. The variances of all quantile sensitivity estimators go up as the probability levels grow up, and in particular, the variances of GLR-2, CGLR-2, CGLRQ-2 go up dramatically when the probability levels grow up from $\alpha=0.7$ to $\alpha=0.9$. The variances of GLR-1, CGLR-1, CGLRQ-1 are smaller than those of FDC(0.1) and $\mathrm{FDC}(0.01)$, and the $\mathrm{CMC}$ and RQMC lead to significant variance reduction.

\section{CONCLUSIONS}

We combine GLR methods in Peng et al. (2018) and Peng et al. (2021) with CMC and RQMC to reduce the variance of quantile sensitivity estimation, and apply the proposed methods to a toy example and a SAN example. In both examples, the GLR method in Peng et al. (2021) leads to a better performance than the GLR method in Peng et al. (2018), and the variance reduction by CMC and RQMC is significant.

However, the variance reduction by RQMC for estimator (2) after smoothing the GLR estimators for two distribution sensitivities by CMC are not as substantial as that for distribution sensitivity estimators themselves (see Peng et al. 2021). The reason may be due to that the quantile estimator by order statistics is discontinuous with respect to the input random variables. This discontinuity issue can be treated by smoothing the empirical distribution function by certain kernel method, which could be a future work.

\section{ACKNOWLEDGMENTS}

This work was supported in part by the National Natural Science Foundation of China (NSFC) under Grants 71901003 and 72022001, by the Air Force Office of Scientific Research under Grant FA95502010211, and by Discovery Grant RGPIN-2018-05795 from NSERC-Canada.

\section{REFERENCES}

Asmussen, S., and P. W. Glynn. 2007. Stochastic Simulation: Algorithms and Analysis, Volume 57. Springer Science \& Business Media. 
Dick, J., and F. Pillichshammer. 2010. Digital Nets and Sequences: Discrepancy Theory and Quasi-Monte Carlo Integration. Cambridge University Press.

Fu, M. C., L. J. Hong, and J.-Q. Hu. 2009. "Conditional Monte Carlo Estimation of Quantile Sensitivities". Management Science 55(12):2019-2027.

Glynn, P. W., Y. Peng, M. C. Fu, and J. Hu. 2021. "Computing Sensitivities for Distortion Risk Measures". INFORMS Journal on Computing, forthcoming.

He, Z., and X. Wang. 2015. "On the Convergence Rate of Randomized Quasi-Monte Carlo for Discontinuous Functions". SIAM Journal on Numerical Analysis 53(5):2488-2503.

Heidergott, B., and W. Volk-Makarewicz. 2016. "A Measure-valued Differentiation Approach to Sensitivity Analysis of Quantiles". Mathematics of Operations Research 41(1):293-317.

Hong, L. J. 2009. "Estimating Quantile Sensitivities". Operations Research 57(1):118-130.

Hong, L. J., and G. Liu. 2009. "Simulating Sensitivities of Conditional Value At Risk". Management Science 55(2):281-293.

Jiang, G., and M. C. Fu. 2015. "Technical Note - On Estimating Quantile Sensitivities Via Infinitesimal Perturbation Analysis". Operations Research 63(2):435-441.

L'Ecuyer, P. 2009. "Quasi-Monte Carlo methods with applications in finance". Finance and Stochastics 42(6):926-938.

L'Ecuyer, P. 2018. "Randomized quasi-Monte Carlo: An introduction for practitioners". Monte Carlo and Quasi-Monte Carlo Methods: MCQMC 2016 (Glynn P. W., Owen A. B. eds.):29-52.

L'Ecuyer, P., and C. Lemieux. 2000. "Variance Reduction via Lattice Rules". Management Science 46(9):1214-1235.

L'Ecuyer, P., and D. Munger. 2012. "Randomized quasi-Monte Carlo: An introduction for practitioners". Monte Carlo and Quasi-Monte Carlo Methods 2010 (Plaskota L., Wozniakowski H. eds.):133-159.

L'Ecuyer, P., and D. Munger. 2016. "Algorithm 958: Lattice builder: A general software tool for constructing rank-1 lattice rules". ACM Transactions on Mathematical Software 42(2):1-30.

Lemieux, C. 2009. Monte Carlo and Quasi-Monte Carlo Method. New York: Springer.

Liu, G., and L. J. Hong. 2009. "Kernel Estimation of Quantile Sensitivities". Naval Research Logistics 56(6):511-525.

Matousek, J. 1998. "On the $L_{2}$-discrepancy for anchored boxes". Journal of Complexity 14(4):527-556.

Nakayama, M. K., Z. T. Kaplan, Y. Li, B. Tuffin, and P. L'Ecuyer. 2020. "Quantile Estimation Via a Combination of Conditional Monte Carlo and Randomized Quasi-Monte Carlo". Proceedings of Winter Simulation Conference:301-312.

Peng, Y., M. C. Fu, P. W. Glynn, and J.-Q. Hu. 2017. "On the asymptotic analysis of quantile sensitivity estimation by Monte Carlo simulation”. Proceedings of Winter Simulation Conference:2336-2347.

Peng, Y., M. C. Fu, B. Heidergott, and H. Lam. 2020. "Maximum Likelihood Estimation By Monte Carlo Simulation: Towards Data-Driven Stochastic Modeling”. Operations Research 68(6):1896-1912.

Peng, Y., M. C. Fu, J. Hu, P. L'Ecuyer, and B. Tuffin. 2021. "Generalized Likelihood Ratio Method for Stochastic Models with Uniform Random Numbers As Inputs". submittted to Operations Research, preprint in https://hal.inria.fr/hal-02652068/document.

Peng, Y., M. C. Fu, J. Hu, and L. Lei. 2019. "Estimating quantile sensitivity for financial models with correlations and jumps". In Proceedings of Winter Simulation Conference, 962-973.

Peng, Y., M. C. Fu, J.-Q. Hu, and B. Heidergott. 2018. "A New Unbiased Stochastic Derivative Estimator for Discontinuous Sample Performances with Structural Parameters”. Operations Research 66(2):487-499.

\section{AUTHOR BIOGRAPHIES}

YIJIE PENG is an Assistant Professor in Guanghua School of Management, with affiliate faculty appointments in the Institute of Artificial Intelligence and National Institute of Health Data Science, all at Peking University. His research interests include stochastic modeling and analysis, simulation optimization, machine learning, data analytics, and healthcare. He is a member of INFORMS and IEEE, and serves 
as an Associate Editor of the Asia-Pacific Journal of Operational Research and the Conference Editorial Board of the IEEE Control Systems Society. His email address is pengyijie@pku.edu.cn.

MICHAEL C. FU holds the Smith Chair of Management Science in the Robert H. Smith School of Business, with a joint appointment in the Institute for Systems Research and affiliate faculty appointment in the Department of Electrical and Computer Engineering, all at the University of Maryland. His research interests include simulation optimization and applied probability. He served as WSC2011 Program Chair, NSF Operations Research Program Director, Management Science Stochastic Models and Simulation Department Editor, and Operations Research Simulation Area Editor. He is a Fellow of INFORMS and IEEE. His e-mail addresses is mfu@umd.edu.

JIAQIAO HU is an Associate Professor in the Department of Applied Mathematics and Statistics at the State University of New York, Stony Brook. He received the BS degree in automation from Shanghai Jiao Tong University, the MS degree in applied mathematics from the University of Maryland, Baltimore County, and the PhD degree in electrical engineering from the University of Maryland, College Park. His research interests include Markov decision processes, applied probability, and simulation optimization. He is currently Department Editor for IISE Transactions and Associate Editor for Operations Research. His email address is jqhu@ams.stonybrook.edu.

PIERRE L'ECUYER is a Professor in the Département d'Informatique et de Recherche Opérationnelle, at the Université de Montréal, Canada. He is a member of CIRRELT and GERAD research centers. His main research interests are random number generation, quasi-Monte Carlo methods, variance reduction, sensitivity analysis and optimization, and stochastic simulation in general. He has published 280 scientific articles, developed stochastic simulation software, and served as a referee for 160 different scientific journals. He received the Lifetime Professional Achievement Award from the INFORMS Simulation Society in 2020. Website:http://www.iro.umontreal.ca/ lecuyer. His e-mail address is lecuyer@iro.umontreal.ca.

BRUNO TUFFIN received his $\mathrm{PhD}$ degree in applied mathematics from the University of Rennes 1 (France) in 1997. Since then, he has been with Inria in Rennes. His research interests include developing Monte Carlo and quasi-Monte Carlo simulation techniques for the performance evaluation of telecommunication systems and telecommunication-related economical models. He is currently Area Editor for INFORMS Journal on Computing and Associate Editor for ACM Transactions on Modeling and Computer Simulation. He has written or co-written three books (two devoted to simulation): Rare event simulation using Monte Carlo methods published by John Wiley \& Sons in 2009, La simulation de Monte Carlo (in French), published by Hermes Editions in 2010, and Telecommunication Network Economics: From Theory to Applications, published by Cambridge University Press in 2014. His email address is bruno.tuffin@inria.fr. 